\title{
A bandwidth correction to the Allegri-Zhang solution for accelerated random vibration testing
}

\author{
Denis Benasciutti , Marta Carlet, and Davide Zanellati \\ University of Ferrara, Department of Engineering, via Saragat 1, 44122, Ferrara, Italy
}

\begin{abstract}
In 2008, Allegri and Zhang published a study [Int. J. Fatigue. 2008, 30(6):967-977] in which they provided an exact analytical solution to the inverse scaling law for accelerated vibration tests of linear systems submitted to stationary Gaussian excitations By combining finite element analysis with multiaxial spectral methods defined in the frequency-domain, their solution generalised the simple inverse power law model suggested in some standards. The solution adopted the "equivalent von Mises stress" multiaxial criterion combined with the narrow-band damage expression. This work aims to propose a bandwidth correction to the original Allegri-Zhang solution to account for the actual spectral bandwidth of the local multiaxial stress. The corrected Allegri-Zhang solution is also extended to another multiaxial spectral method, namely the "Projection-by-Projection" criterion. A numerical example is finally discussed, in which the corrected solution is applied to an L-shaped beam submitted to random accelerations.
\end{abstract}

\section{Introduction}

Mechanical systems are often exposed to vibrations, which induce randomly varying stresses that may be responsible for fatigue damage and even lead to failures. To verify the component service life in the laboratory, it is customary to perform accelerated vibratory tests in which the excitation level is considerably higher than the actual one, which allows the testing time to be greatly shortened compared to the actual life of the system (which may be thousands of hours). Inverse power scaling laws are commonly used to correlate the time to failure in the accelerated laboratory test to the life estimated under real excitations. The following simple power law model is commonly used [1-3]:

$$
\frac{T_{\mathrm{s}}}{T_{\mathrm{r}}}=\left(\frac{\bar{g}_{\mathrm{r}}}{\bar{g}_{\mathrm{s}}}\right)^{\alpha}
$$

where $T_{\mathrm{r}}$ is the time to failure that a mechanical system is expected to survive in the actual operative environment, $T_{\mathrm{s}}$ is the time to failure in the laboratory test, $\bar{g}_{\mathrm{r}}$ and $\bar{g}_{\mathrm{s}}$ are the root mean square (RMS) values of acceleration in the actual and laboratory environments, respectively. In the original derivation of Eq. (1), the exponent $\alpha$ represents the inverse slope of the S-N fatigue curve. In practical applications, however, $\alpha$ is often treated as an empirical factor (ranging from 5 to 8) that summarises all the uncertainties and approximations in Eq. (1) and its value is often adjusted based on previous experience [3].

Due to its simplicity, the scaling law (1) has been largely used in industry, especially at the design stage, and it is currently included in MIL Standard 810 [1]. Such a simple law, however, only provide a rough approximation of the complex stresses really experienced by a structure under a random input. For example, Eq. (1) neglects the actual shape of the acceleration PSD, which is only synthesised by its RMS value. It also ignores the structure dynamic behaviour, which may directly affect the output stress.

In 2008, Allegri and Zhang published a research study [3] in which they contextualized the scaling law (1) in a more general theory based on finite element analysis and multiaxial spectral methods defined in the frequencydomain through a Power Spectral Density (PSD) matrix - their theory adopted the "equivalent von Mises stress" (EVMS) criterion. The Allegri-Zhang's study derived an exact solution of a general scaling law model, of which the empirical relationship (1) is only a simplified case.

Despite its novelty, their solution seems not to have received so much attention, nor was it further applied in practical case studies. In Allegri-Zhang (A-Z) approach, though, some issues are worth to be investigated, as for example the spectral bandwidth of the multiaxial stress state, the accuracy of the EVMS criterion, or the possibility to implement other multiaxial criteria. With these premises, this work will investigate the A-Z solution with the aim to:

i) propose a modified solution that includes the spectral bandwidth of the local stress state, which was ignored in the original approach;

ii) discuss the role of S-N parameters in EVMS criterion and, accordingly, to suggest possible corrections;

iii) extend the A-Z solution to the "Projection-byProjection" multiaxial criterion. 
A simple numerical example will be finally discussed, in which the obtained scaling-law solutions are applied to an L-shaped beam submitted to random accelerations imitating the real and accelerated laboratory environments. A comparison with the simple scaling law (1) will be investigated by analysing the results for different acceleration PSD shapes.

\section{Spectral description of uniaxial and multiaxial random stresses}

Let $x(t)$ be a uniaxial zero-mean stationary and Gaussian random stress with two-sided auto-PSD $S(f)$, which is characterised by the set of spectral moments [4]:

$$
\lambda_{\mathrm{n}}=\int_{-\infty}^{\infty}|f|^{\mathrm{n}} S(f) \mathrm{d} f \quad n=1,2, \mathrm{~K}
$$

The variance is $\lambda_{0}=\operatorname{Var}(x(t))$ and the root mean square (RMS) value is $\bar{x}=\sqrt{\lambda_{0}}$ [4]. The frequency of mean upcrossings and the peaks rate are, respectively [4]:

$$
v_{0}=\sqrt{\lambda_{2} / \lambda_{0}} \quad ; \quad v_{\mathrm{p}}=\sqrt{\lambda_{4} / \lambda_{2}}
$$

The following bandwidth parameters will be considered:

$$
\alpha_{1}=\frac{\lambda_{1}}{\sqrt{\lambda_{0} \lambda_{2}}} \quad, \quad \alpha_{2}=\frac{\lambda_{2}}{\sqrt{\lambda_{0} \lambda_{4}}}
$$

where $0 \leq \alpha_{\mathrm{m}} \leq 1$ and $\alpha_{1} \geq \alpha_{2}$. Note that $\alpha_{2}=v_{0} / v_{\mathrm{p}}$ ("regularity index"). Two limiting cases exist: a narrow-band PSD that has $\alpha_{1} \rightarrow 1, \alpha_{2} \rightarrow 1$, a wide-band PSD that has $\alpha_{1}<1$, $\alpha_{2}<1$ (e.g., a white noise spectrum has $\alpha_{2} \cong 0.745$ ). If $\alpha_{2}<<1$, the signal has many small oscillations between two successive zero up-crossings $\left(v_{0}<<v_{\mathrm{p}}\right)$.

The previous frequency-domain description can be extended to a multiaxial stationary random stress. For the sake of simplicity, equations will be presented only for a biaxial stress, although the same formalism applies to a three-dimensional stress. Let $\mathbf{x}(t)=\left(\sigma_{\mathrm{xx}}(t), \sigma_{\mathrm{yy}}(t), \tau_{\mathrm{xy}}(t)\right)$ be a biaxial stress ( $\sigma$ is normal stress, $\tau$ shear stress), characterised by the Hermitian PSD matrix:

$$
\mathbf{S}_{\sigma}(f)=\left[\begin{array}{ccc}
S_{\mathrm{xx}}(f) & S_{\mathrm{xx}, \mathrm{yy}}(f) & S_{\mathrm{xx}, \mathrm{xy}}(f) \\
S_{\mathrm{xx}, \mathrm{yy}}^{*}(f) & S_{\mathrm{yy}}(f) & S_{\mathrm{yy}, \mathrm{xy}}(f) \\
S_{\mathrm{xx}, \mathrm{xy}}^{*}(f) & S_{\mathrm{yy}, \mathrm{xy}}^{*}(f) & S_{\mathrm{xy}}(f)
\end{array}\right]
$$

in which the diagonal terms are auto-PSDs and the outof-diagonal terms are cross-PSDs (complex functions).

\section{Spectral methods and fatigue life}

For a narrow-band uniaxial stress of time duration $T$, the fatigue damage is $[5,6]$ :

$$
D_{\mathrm{NB}}=\frac{T v_{0}}{C}\left(\sqrt{2 \lambda_{0}}\right)^{\mathrm{b}} \Gamma\left(1+\frac{b}{2}\right)
$$

where $C, b$ are the parameters of the S-N curve $s^{\mathrm{b}} N=C$. The coefficient $C=s_{\mathrm{A}}^{\mathrm{b}} N_{\mathrm{A}}$ relates to the reference stress amplitude $s_{\mathrm{A}}$ at $N_{\mathrm{A}}=2 \times 10^{6}$ cycles.

The time to failure follows by settling $D_{\mathrm{NB}}=D_{\text {cr }}$ (where $D_{\text {cr }}$ is a critical damage value):

$$
T_{\mathrm{f}, \mathrm{NB}}=\frac{C D_{\mathrm{cr}}}{v_{0}\left(\sqrt{2 \lambda_{0}}\right)^{\mathrm{b}} \Gamma\left(1+\frac{b}{2}\right)}
$$

In the narrow-band case Eq. (7) is exact, while in the wide-band case it is largely conservative and needs to be corrected by accounting for the actual PSD bandwidth $[5,6]$. A very simple approach is to introduce a correction factor $\rho \leq 1$ in Eq. (7), so that for a wide-band stress:

$$
T_{\mathrm{f}}=\frac{C D_{\mathrm{cr}}}{\rho v_{0}\left(\sqrt{2 \lambda_{0}}\right)^{\mathrm{b}} \Gamma\left(1+\frac{b}{2}\right)}
$$

Factor $\rho$ depends on the PSD shape and it is a function of spectral moments and bandwidth parameters. In the narrow-band case $\rho \rightarrow 1$, while in the wide-band case $\rho \rightarrow 0$ (factor $\rho$ then gives an indirect measure of the spectral width of a PSD). Several methods adopt such a correction factor approach, as the "TB method" $[5,6]$ :

$$
\rho=\left[q+(1-q) \alpha_{2}^{\mathrm{b}-1}\right]
$$

where $q=q\left(\alpha_{1}, \alpha_{2}\right)$ is a coefficient depending on $\alpha_{1}, \alpha_{2}$ (its analytical expression can be found in $[5,6]$ ).

Other methods also exist (e.g. Dirlik) which replace the single factor $\rho$ with more elaborated expressions [6]. However, to keep things simple and without any loss of generality, it will be assumed that bandwidth effects are summarised by factor $\rho$ and that the time to failure $T_{\mathrm{f}}$ for a wide-band uniaxial stress is estimated by Eq. (8).

With a multiaxial stress, a suitable criterion needs to be introduced for estimating the time to failure. A criterion largely used is that based on the "Equivalent Von Mises Stress" (EVMS) $\sigma_{\mathrm{eq}}(t)$, which is a zero-mean Gaussian uniaxial stress, described by the following PSD:

$$
S_{\mathrm{eq}}(f)=S_{\mathrm{xx}}(f)+S_{\mathrm{yy}}(f)-\operatorname{Re}\left[S_{\mathrm{xx}, \mathrm{yy}}(f)\right]+3 S_{\mathrm{xy}}(f)
$$

In matrix form ("Tr" is the trace operator):

$$
S_{\mathrm{eq}}(f)=\operatorname{Tr}\left\{\mathbf{Q S}_{\sigma}(f)\right\}, \quad \mathbf{Q}=\left[\begin{array}{ccc}
1 & -0.5 & 0 \\
-0.5 & 1 & 0 \\
0 & 0 & 3
\end{array}\right]
$$

The RMS value of $\sigma_{\text {eq }}(t)$ is $\bar{\sigma}_{\text {eq }}=\sqrt{\lambda_{0, \text { eq }}}$, where $\lambda_{\text {eq }}$ is the zero-order moment of $S_{\mathrm{eq}}(f)$. Note that in general $S_{\text {eq }}(f)$ is wide-band, which then requires that Eq. (8) is applied to estimate the fatigue life. 


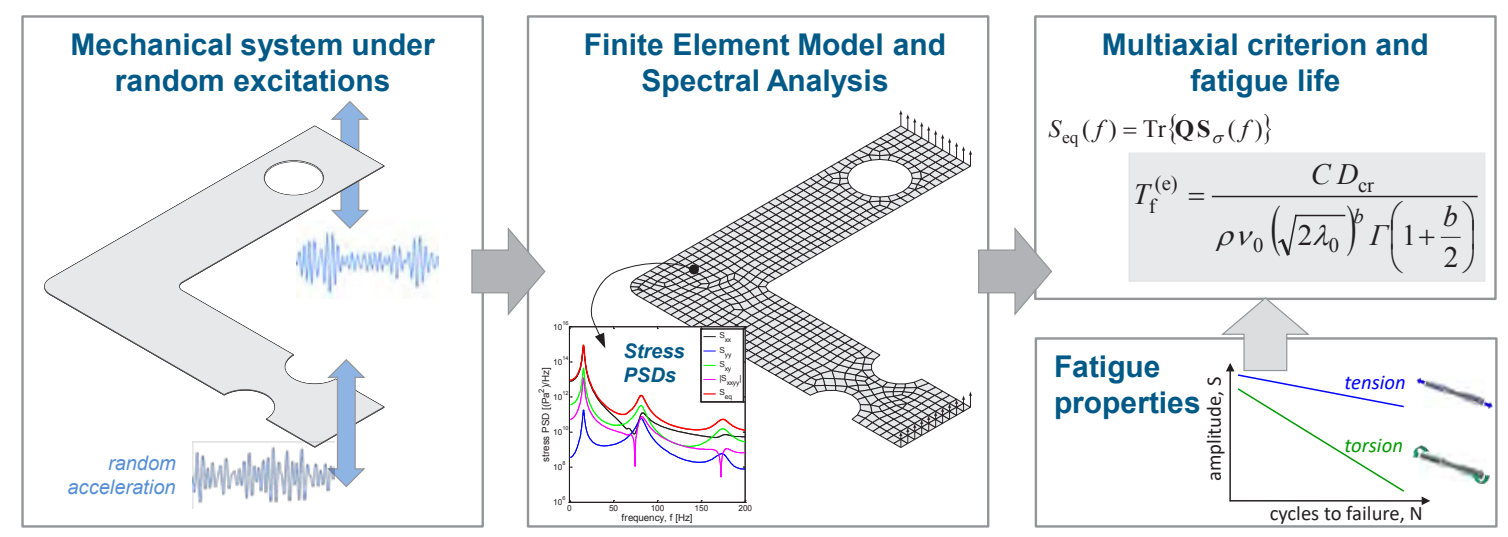

Fig. 1. Analysis steps (for both "real" and "lab" environment): finite element model, spectral analysis to compute the stress PSDs at each element, multiaxial spectral method to estimate the time to failure.

\section{Accelerated tests and scaling law}

\subsection{Allegri-Zhang (A-Z) original solution}

Assume that a mechanical system is subjected to a random base acceleration. The finite element method can profitably be exploited to evaluate the structure dynamic response and the stress PSD matrix $\mathbf{S}_{\sigma}^{(\mathrm{e})}(f)$ in each element (e) of the model (Fig. 1). For a plane stress state, $\mathbf{S}_{\sigma}^{(\mathrm{e})}(f)$ is a $3 \times 3$ matrix, see Eq. (5).

In the A-Z approach [3], the exact scaling law was derived by considering two main assumptions. The first one was to use the EVMS criterion, Eq. (11), to transform $\mathbf{S}_{\sigma}^{(\mathrm{e})}(f)$ of a multiaxial stress into $S_{\mathrm{eq}}^{(\mathrm{e})}(f)$ for the equivalent stress $\sigma_{\mathrm{eq}}(t)$.

The second assumption was the choice of the statistical parameter of $\sigma_{\mathrm{eq}}(t)$ to construct a stress-life relationship. The stress level was quantified by $\bar{\sigma}_{\text {eq }}$, i.e. the RMS value of $\sigma_{\mathrm{eq}}(t)$. The number of cycles to failure, instead, was measured by the number of zero up-crossings $N_{0}$ observed in $\sigma_{\mathrm{eq}}(t)$. For a stationary stress $N_{0}=v_{0 \mathrm{eq}} \cdot T_{\mathrm{f}}$, where $T_{\mathrm{f}}$ is the fatigue life (in seconds) and $v_{0 \text { eq }}$ the rate of zero up-crossings for $\sigma_{\text {eq }}(t)$. An S-N type equation $\bar{\sigma}_{\text {eq }}^{\mathrm{b}} N_{0}=C$ was then introduced and the fatigue life estimated as:

$$
T_{\mathrm{f}}=\frac{C}{v_{0 \mathrm{eq}} \bar{\sigma}_{\mathrm{eq}}^{\mathrm{b}}}
$$

Although $b, C$ virtually represent the parameters of the constant amplitude S-N curve, their value may change under a random stress, as also pointed out in [3] (this issue will be further discussed in Section 6).

To derive a scaling law, Eq. (12) has to be applied twice: first for estimating the fatigue life $T_{\mathrm{f}, \mathrm{r}}$ of the mechanical system exposed to the "real" environment (actual excitation), secondly for assessing the fatigue life $T_{\mathrm{f}, \mathrm{s}}$ in the laboratory accelerated test. The following ratio of time durations then results:

$$
\frac{T_{\mathrm{f}, \mathrm{s}}}{T_{\mathrm{f}, \mathrm{r}}}=\frac{v_{0 \mathrm{eq}, \mathrm{r}}}{v_{0 \mathrm{eq}, \mathrm{s}}}\left(\frac{\bar{\sigma}_{\mathrm{eq}, \mathrm{r}}}{\bar{\sigma}_{\mathrm{eq}, \mathrm{s}}}\right)^{\mathrm{b}}
$$

where $v_{0 \text { eq,r }}, \bar{\sigma}_{\text {eq,r }}=\sqrt{\lambda_{0 \text { eq,r }}}$ are the spectral parameters of the equivalent von Mises stress in the "real" environment, while $v_{0 \mathrm{eq}, \mathrm{s}}, \bar{\sigma}_{\mathrm{eq}, \mathrm{s}}=\sqrt{\lambda_{\text {0eq, } \mathrm{s}}}$ are those for the laboratory experiment. Being a scaling law, Eq. (13) only depends on the slope $b$, but not on the coefficient $C$. Expanding Eq. (13) in terms of spectral moments yields:

$$
\frac{T_{\mathrm{f}, \mathrm{s}}}{T_{\mathrm{f}, \mathrm{r}}}=\sqrt{\frac{\lambda_{2 \mathrm{eq}, \mathrm{r}}}{\lambda_{2 \mathrm{eq}, \mathrm{s}}}}\left(\frac{\lambda_{0 \mathrm{eq}, \mathrm{r}}}{\lambda_{0 \mathrm{eq}, \mathrm{s}}}\right)^{\frac{\mathrm{b}-1}{2}}
$$

Note that Eq. (14) only depends on two spectral moments $\lambda_{0 \text { eq }}$ and $\lambda_{2 \text { eq }}$ of $S_{\text {eq }}^{(\mathrm{e})}(f)$. By writing explicitly the definition of such spectral moments, see Eq. (2), the previous expression returns the final equation in [3]:

$\left.\frac{T_{\mathrm{f}, \mathrm{s}}^{(\mathrm{e})}}{T_{\mathrm{f}, \mathrm{r}}^{(\mathrm{e})}}=\sqrt{\frac{\operatorname{Tr}\left[\mathbf{Q} \int_{0}^{\infty} f^{2} \mathbf{S}_{\sigma, \mathrm{r}}^{(\mathrm{e})}(f) \mathrm{d} f\right]}{\operatorname{Tr}\left[\mathbf{Q} \int_{0}^{\infty} f^{2} \mathbf{S}_{\sigma, \mathrm{s}}^{(\mathrm{e})}(f) \mathrm{d} f\right]}\left\{\frac{\operatorname{Tr}\left[\mathbf{Q} \int_{0}^{\infty} \mathbf{S}_{\sigma, \mathrm{r}}^{(\mathrm{e})}(f) \mathrm{d} f\right]}{\operatorname{Tr}\left[\mathbf{Q} \int_{0}^{\infty} \mathbf{S}_{\sigma, \mathrm{s}}^{(\mathrm{e})}(f) \mathrm{d} f\right]}\right.}\right\}^{\frac{\mathrm{b}^{(\mathrm{e})}-1}{2}}$

The superscript (e) is reintroduced to remind that the quantities in Eq. (15) refer to element (e) in the finite element model. Also the slope $b^{(\mathrm{e})}$ depends on the material which the element is made of.

Equation (15) is the original $\mathrm{A}-\mathrm{Z}$ solution and it represents the exact scaling law for linear systems undergoing Gaussian random accelerations.

Allegri and Zhang also demonstrated that Eq. (15) returns exactly the scaling law (1) in the special case in which the acceleration PSD for the "lab" environment is simply scaled with respect to that characterising the "real" environment [3].

It has to be emphasised that the scaling law (15) is based on the hypothesis to take $N_{0}$ as a measure of the number of rainflow cycles, which is exact only for a narrow-band 
stress (where $v_{0} \cong v_{\mathrm{p}}$ ). In a wide-band stress, it is $N_{0}<N_{\mathrm{p}}$, where $N_{\mathrm{p}}$ is the number of peaks, which in turn is coincident with the number of rainflow cycles (in rainflow counting, a cycle is attached to each peak). In a wide-band stress, $N_{0}$ would then underestimate the actual number of rainflow cycles.

This conclusion is also confirmed by noting that the relationship (14) only involves two spectral moments $\lambda_{0}$ and $\lambda_{2}$, which implies that it does not accounts for the actual spectral bandwidth of $\sigma_{\mathrm{eq}}(t)$, i.e. it provides the same result for any type of narrow-band and wide-band processes sharing the same values of $\lambda_{0}$ and $\lambda_{2}$. For example, one may construct an infinite set of wide-band spectra $S_{\text {eq }}^{(\mathrm{e})}(f)$ that have common values of $\lambda_{0}$ and $\lambda_{2}$, but have different values of other spectral moments (e.g. $\lambda_{1}$ and $\lambda_{4}$ ). Such spectra would have identical values of $v_{0 \text { eq }}, \bar{\sigma}_{\text {eq }}$, and also the same value of $T_{\mathrm{f}}$ from Eq. (12).

These arguments motivate the attempt to introduce a bandwidth correction to the A-Z solution, as explained in the next section.

\subsection{Bandwidth correction of A-Z solution}

The hypothesis in A-Z solution that $\sigma_{\text {eq }}(t)$ is considered to be always narrow-band is confirmed by noting that Eq. (13) can also be obtained more directly by applying the narrow-band expression (7) to the "real" and "lab" environments.

To account for the actual bandwidth of $\sigma_{\mathrm{eq}}(t)$, the wideband solution (8) has to be used in place of Eq. (7). In the "real" environment, the time to failure is:

$$
T_{\mathrm{f}, \mathrm{r}}=\frac{C D_{\mathrm{cr}}}{\rho_{\mathrm{eq}, \mathrm{r}} \nu_{0 \mathrm{eq}, \mathrm{r}}\left(\sqrt{2 \lambda_{0 \mathrm{eq}, \mathrm{r}}}\right)^{\mathrm{b}} \Gamma\left(1+\frac{b}{2}\right)}
$$

where subscript $r$ stands for "real". As before, symbols $v_{0 \text { eq, },}, \bar{\sigma}_{\text {eq,r }}=\sqrt{\lambda_{0 \text { eq,r }}}$ identify the zero up-crossings and RMS value of $\sigma_{\mathrm{eq}}(t)$, whereas $\rho_{\mathrm{eq}, \mathrm{r}}$ is the bandwidth correction factor (9) according to the TB method. For the "simulation" ("lab") environment, the time to failure $T_{\mathrm{f}, \mathrm{s}}$ is given by an expression identical to Eq. (16), in which symbols $v_{0 \text { eq, } s,}, \bar{\sigma}_{\text {eq,s }}=\sqrt{\lambda_{\text {leq }, \mathrm{s}}}, \rho_{\text {eq, } \mathrm{s}}$ are used:

$$
T_{\mathrm{f}, \mathrm{s}}=\frac{C D_{\mathrm{cr}}}{\rho_{\text {eq,s }} \nu_{0 \text { eq }, \mathrm{s}}\left(\sqrt{2 \lambda_{0 \mathrm{eq}, \mathrm{s}}}\right)^{\mathrm{b}} \Gamma\left(1+\frac{b}{2}\right)}
$$

The scaling law is constructed as the ratio:

$$
\frac{T_{\mathrm{f}, \mathrm{s}}}{T_{\mathrm{f}, \mathrm{r}}}=\frac{\rho_{\text {eq,r }}}{\rho_{\text {eq,s }}} \frac{v_{0 \mathrm{eq}, \mathrm{r}}}{v_{0 \mathrm{eq}, \mathrm{s}}}\left(\frac{\bar{\sigma}_{\mathrm{eq}, \mathrm{r}}}{\bar{\sigma}_{\mathrm{eq}, \mathrm{s}}}\right)^{\mathrm{b}}
$$

which generalises the original A-Z solution (13) by introducing a spectral bandwidth correction factor $\rho$ for the PSD of the equivalent stress. Note that, in general, $\rho_{\text {eq, }} \neq \rho_{\text {eq,s. }}$ In fact, if the same structure is excited by "real" and "lab" acceleration PSDs with different shapes, it may be expected that different stress PSDs will also be obtained. For example, if the "real" acceleration is narrow-band, the output stress is close to narrow-band. Instead, if the "lab" acceleration is wide-band, it is more likely that at some node the stress PSD is more wideband. The condition $\rho_{\text {eq, }}=\rho_{\text {eq, s }}$ occurs only if the "real" and "lab" acceleration PSDs are exactly scaled.

Similarly to the A-Z solution (15), the ratio of time durations can be further manipulated to obtain:

$\frac{T_{\mathrm{f}, \mathrm{s}}^{(\mathrm{e})}}{T_{\mathrm{f}, \mathrm{r}}^{(\mathrm{e})}}=\sqrt{\frac{\rho_{\mathrm{eq}, \mathrm{r}} \operatorname{Tr}\left[\mathbf{Q} \int_{0}^{\infty} f^{2} \mathbf{S}_{\sigma, \mathrm{r}}^{(\mathrm{e})}(f) \mathrm{d} f\right]}{\rho_{\mathrm{eq}, \mathrm{s}} \operatorname{Tr}\left[\mathbf{Q} \int_{0}^{\infty} f^{2} \mathbf{S}_{\sigma, \mathrm{s}}^{(\mathrm{e})}(f) \mathrm{d} f\right]}}\left\{\frac{\operatorname{Tr}\left[\mathbf{Q} \int_{0}^{\infty} \mathbf{S}_{\sigma, \mathrm{r}}^{(\mathrm{e})}(f) \mathrm{d} f\right]}{\operatorname{Tr}\left[\mathbf{Q} \int_{0}^{\infty} \mathbf{S}_{\sigma, \mathrm{s}}^{(\mathrm{e})}(f) \mathrm{d} f\right]}\right\}^{\frac{\mathrm{b}^{(\mathrm{e})}-1}{2}}$

in which superscript (e) reminds that quantities refer to element (e) in the finite element model.

Equation (19) is the bandwidth correction of the original A-Z solution (15). Compared to Eq. (15), the two factors $\rho_{\text {eq,r },}, \rho_{\text {eq,s }}$ appear. If in each element (e) the equivalent stress $\sigma_{\mathrm{eq}}(t)$ is always narrow-band, $\rho_{\mathrm{eq}, \mathrm{r}}=\rho_{\mathrm{eq}, \mathrm{s}}=1$ and Eq. (19) converges to the original A-Z scaling law (15).

\section{Numerical example}

The purpose of this numerical example is to show the practical application of the analytical scaling laws discussed in the previous sections. The example considers an L-shaped beam excited by a base random acceleration. The beam geometry imitates that one already proposed in [7], although some dimensions have been slightly changed to enhance the stress concentration effect at the hole and two lateral notches.

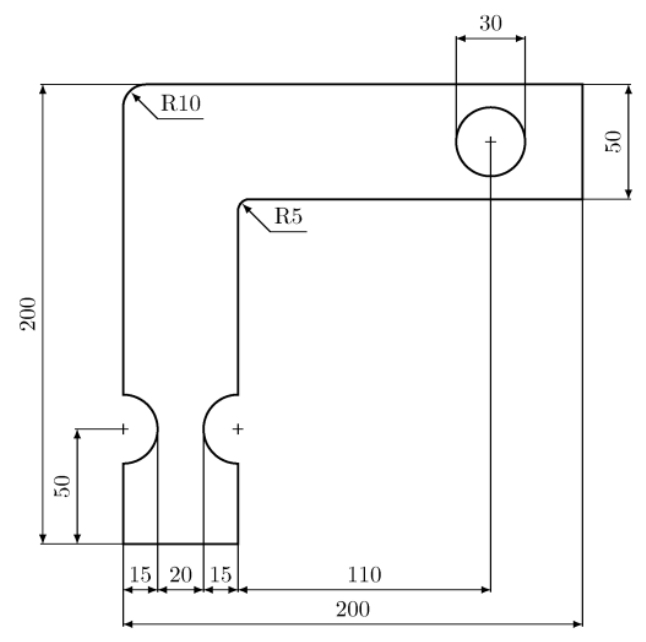

Fig. 2. Beam dimensions (in $\mathrm{mm}$ ). Thickness is $0.5 \mathrm{~mm}$.

The specimen is clamped at both ends, where a random acceleration is imposed along the direction normal to the specimen plane. The input accelerations applied at the two clamped ends are assumed to be fully correlated (their cross-PSD is different from zero). 
The beam is made of steel; its tension S-N curve has parameters (taken from [7]) $b=9.82, C=4.0641 \times 10^{88}$ (the reference strength amplitude is $s_{\mathrm{A}}=241 \mathrm{MPa}$ ).

A 'shell' finite element model is used to discretise the beam (elements have an average size of $5 \mathrm{~mm}$, resulting in 641 elements and 735 nodes), see Fig. 3.

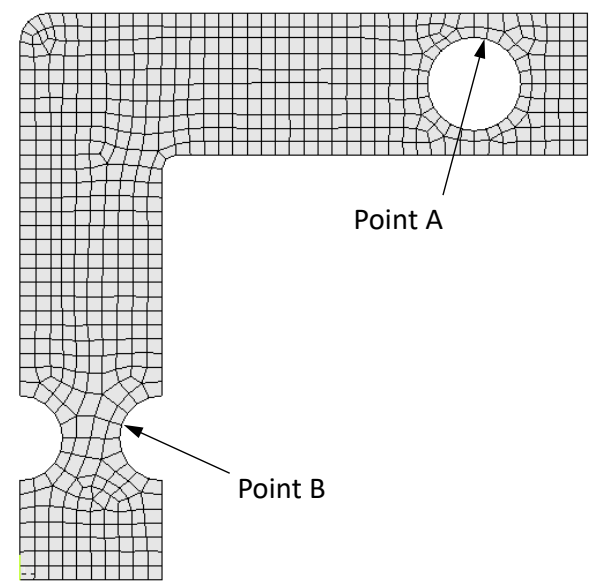

Fig. 3. Finite element model (shell elements).

In the finite element simulation (Fig. 1), a frequencydomain spectrum analysis is carried out to determine the natural frequencies and the stress PSDs at each node. Stress spectra are next processed to determine the time to failure used in the inverse scaling laws discussed in the previous sections. The whole numerical analysis is performed by software ANSYS with APDL language, which is also used to implement the scaling laws.

A modal analysis returns the following first natural frequencies: $f_{1}=15.9 \mathrm{~Hz}, f_{2}=67.1 \mathrm{~Hz}, f_{3}=81.9 \mathrm{~Hz}$, $f_{4}=175.1 \mathrm{~Hz}, f_{5}=178.9 \mathrm{~Hz}$. A harmonic analysis is also carried out to determine the frequency response function and the contribution of each mode, see Fig. 4. The figure clearly shows the peaks at each resonant frequency (two of them are less pronounced).

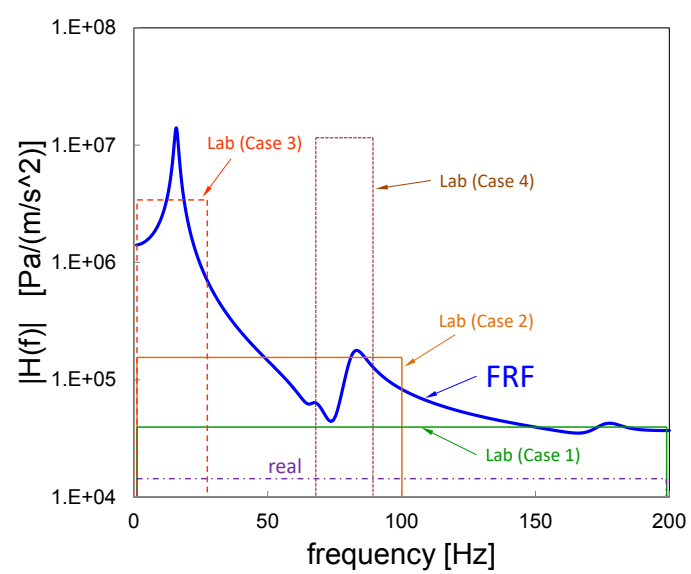

Fig. 4. Frequency response function of the L-beam. The acceleration PSDs (Case 1 to 4) are also shown (qualitatively).

In order to imitate the application of the inverse scaling laws, the PSD of the input acceleration has to be defined for both the "real" and "laboratory" environments.
Since the purpose of this example is only to demonstrate the practical application of scaling laws (without any claim to provide results of direct practical use), the acceleration PSD is idealised as a band-limited rectangular spectrum.

For the "real" environment, a rectangular PSD from 1 to $200 \mathrm{~Hz}$ is chosen; its RMS value $\bar{g}_{\mathrm{r}}=2.8833 \mathrm{~g}(g=9.81$ $\mathrm{m} / \mathrm{s}^{2}$ is the gravity acceleration) is so adjusted in order that, in numerical simulations, the beam has a "real" time duration $T_{\mathrm{r}}=10$ years $\left(3.1536 \cdot 10^{8}\right.$ seconds $)$ in the most stressed point.

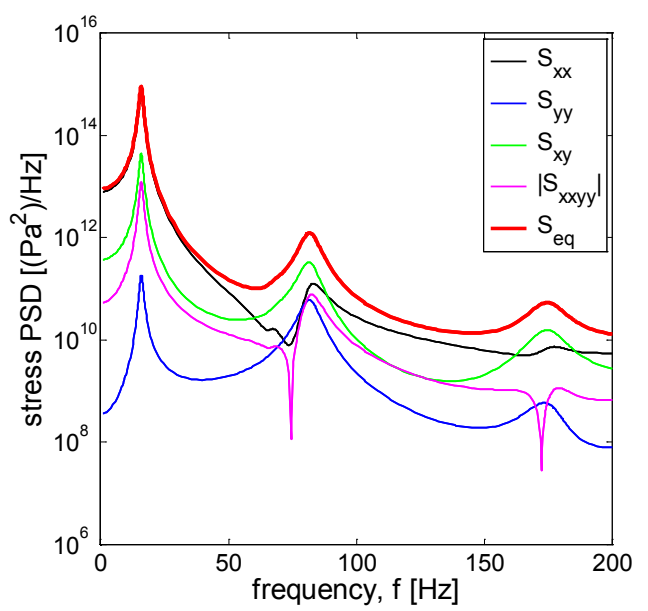

Fig. 5. PSDs of stress components in point A.

Fig. 5 displays the auto- and cross-PSD of the biaxial stress calculated at point A, for the "real" case. The figure highlights how the rectangular PSD of input acceleration is filtered out, resulting in stress PSDs peaked at resonance frequencies. The figure also plots the power spectrum $S_{\text {eq }}(f)$ of the equivalent stress, which is not exactly narrow-band, as it has spectral parameters $\alpha_{1}=0.924, \alpha_{2}=0.447$.

For the "lab" environment, a target time duration of $T_{\mathrm{s}, 0}=1$ day ( 86400 seconds) is taken as the period to be achieved in an hypothetical accelerated testing. The resulting scaling ratio is $\left(T_{\mathrm{s}, 0} / T_{\mathrm{r}}\right)=0.274 \cdot 10^{-3}$.

Also the "lab" acceleration PSD is idealised as a rectangular (band-limited) spectrum. Four different cases are distinguished (see Table 1), based on the following frequency ranges:

- Case 1: rectangular PSD from 1 to $200 \mathrm{~Hz}$ (same shape as the "real" spectrum);

- Case 2: rectangular PSD from 1 to $100 \mathrm{~Hz}$;

- Case 3: rectangular PSD from 1 to $30 \mathrm{~Hz}$;

- Case 4: rectangular PSD from 70 to $90 \mathrm{~Hz}$.

All the previous PSDs share the same value $\bar{g}_{\mathrm{s}}=6.6473 \mathrm{~g}$ of acceleration RMS, which is determined through Eq. (1) by targeting the laboratory time duration $T_{\mathrm{s}, 0}=1$ day and assigning the specifications $T_{\mathrm{r}}=10$ years, $\bar{g}_{\mathrm{r}}=2.8833 \mathrm{~g}$ for the "real" environment (the exponent in Eq. (1) is taken equal to the S-N slope $\alpha=b=9.82$ ).

Fig. 4 compares (qualitatively) the four PSD types to the frequency response function. Being the RMS value the same for all spectra, the height of each rectangle changes depending on the frequency range of each spectrum. 
Simulation results of several test cases are summarised from Fig. 6 to Fig. 9. For the Case 1 acceleration PSD, Fig. 6 displays the RMS value $\bar{\sigma}_{\text {eqs }}$ of the equivalent stress, while Fig. 7 shows the corresponding distribution of the $\operatorname{logarithm} \log \left(T_{\mathrm{s}}\right)$ of the time duration $T_{\mathrm{s}}$ computed in each finite element by Eq. (17).

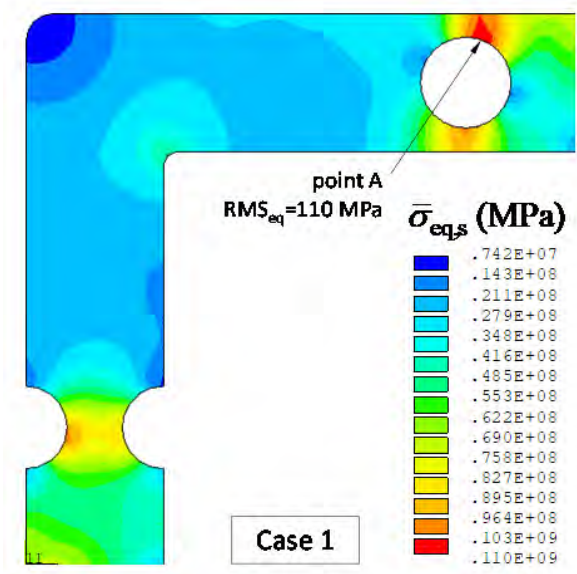

Fig. 6. Distribution of RMS of eq. stress $\sigma_{\text {eq }}$ (Case 1).

The highest RMS value (110 MPa) occurs at point A, where also the shortest time duration $T_{\mathrm{s}}=86400$ seconds is obtained. This time duration exactly matches the target value $T_{\mathrm{s}, 0}$ predicted by Eq. (1). This result confirms that if "lab" and "real" PSDs of the input acceleration have the same shape and are simply scaled, both scaling laws (1) and (19) return the same result. This also holds true for the ratio $\left(T_{\mathrm{s}} / T_{\mathrm{r}}\right)$ in each node of the model, which is always equal to the ratio $\left(T_{\mathrm{s}, 0} / T_{\mathrm{r}}\right)=0.274 \cdot 10^{-3}$.

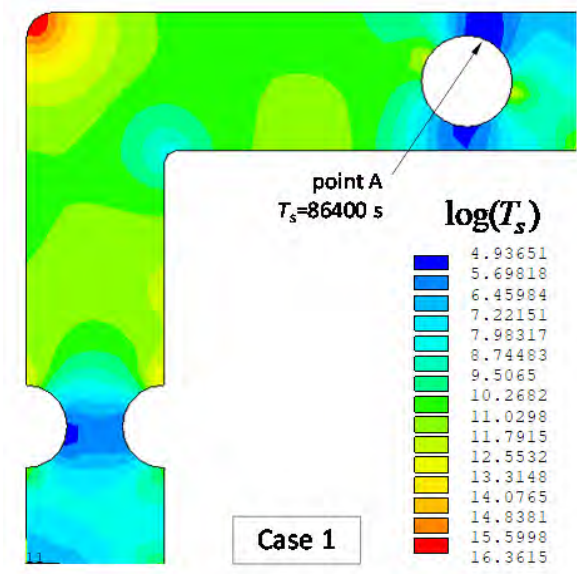

For the Case 2, the distribution of $\log \left(T_{\mathrm{s}}\right)$ is shown in Fig. 8. The shortest fatigue life $T_{\mathrm{s}, \mathrm{A}}=2890$ seconds still occurs at point $\mathrm{A}$, where the highest RMS value (156 $\mathrm{MPa}$ ) is also identified.

Surprisingly, now the value $T_{\mathrm{s}, \mathrm{A}}$ returned by Eq. (17) through numerical analysis is much shorter than the target $T_{\mathrm{s}, 0}$ estimated by Eq. (1). Differently from Case 1, in this Case 2 in which "lab" and "real" acceleration PSDs are not simply scaled, a disagreement is observed in the time scaling estimated from Eq. (1) and Eq. (19).

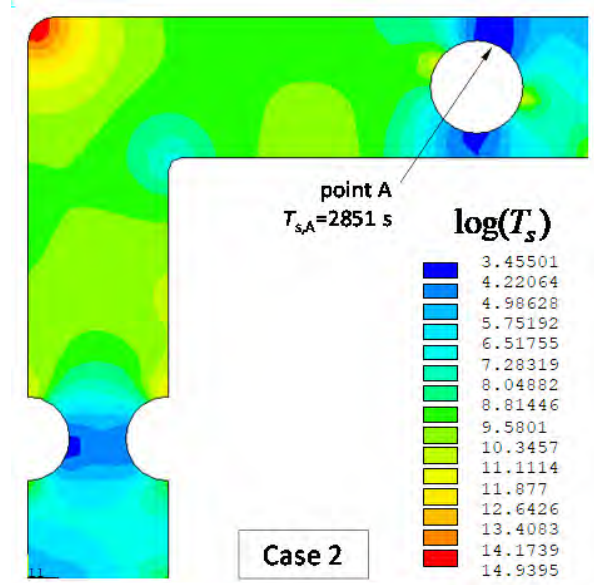

Fig. 8. Distribution of $\log \left(T_{\mathrm{s}}\right)($ Case 2$)$.

This disagreement can be explained by considering that the simple scaling law model (1) provides only a rough approximation of the relationship of the system response in the real and laboratory environments.

First, it takes the acceleration RMS value as the only one parameter synthesising the input acceleration spectrum (the actual spectrum shape and frequency range are not considered). Secondly, the scaling law model (1) also ignores the actual system dynamic response (especially the coupling between modes) and the resulting multiaxial state of stress in each part of the system. On the other hand, these aspects are correctly evaluated by the Allegri-Zhang model in its original as well as modified version.

This behaviour can be explained by observing that the acceleration PSD in Case 2 (ranging in $1 \div 100 \mathrm{~Hz}$ ) has double the height of Case 1 (which ranges in $1 \div 200 \mathrm{~Hz}$ ) and it is more concentrated towards the first natural frequency. This makes the contribution of the first vibration mode enhance the RMS of the output stress.

Fig. 7. Distribution of $\log \left(T_{\mathrm{s}}\right)($ Case 1$)$.

Table 1. Comparison of simulation results for the "real" and "lab" environments.

\begin{tabular}{|c|c|c|c|c|c|}
\hline $\begin{array}{c}\text { Type of } \\
\text { PSD }\end{array}$ & $\begin{array}{c}\text { Acceleration } \\
\text { RMS }\end{array}$ & $\boldsymbol{T}_{\mathbf{s}, \mathbf{0}}$ from Eq. (1) & RMS of eq. stress, $\overline{\boldsymbol{\sigma}}_{\mathbf{e q}}$ (MPa) & $\begin{array}{c}\boldsymbol{T}_{\mathbf{s}} \text { from Eq. (19) - } \\
\text { numerical analysis }\end{array}$ & $\begin{array}{c}\text { New } \\
\text { exponent } \boldsymbol{\alpha}\end{array}$ \\
\hline “real” & $2.8833 g$ & - & 47.7 (in A) & $315360013 \mathrm{~s}$ & - \\
\hline Case 1 & $6.6473 g$ & 1 day $(86400 \mathrm{~s})$ & 110 (in A) & $86400 \mathrm{~s}$ & 9.82 \\
\hline Case 2 & $6.6473 g$ & 1 day $(86400 \mathrm{~s})$ & 156 (in A) & $2851 \mathrm{~s}$ & 13.9 \\
\hline Case 3 & $6.6473 g$ & 1 day $(86400 \mathrm{~s})$ & 287 (in A) & $8.6 \mathrm{~s}$ & 20.9 \\
\hline Case 4 & $6.6473 g$ & 1 day $(86400 \mathrm{~s})$ & 43 (in B) & $17419281 \mathrm{~s}$ & 3.47 \\
\hline
\end{tabular}




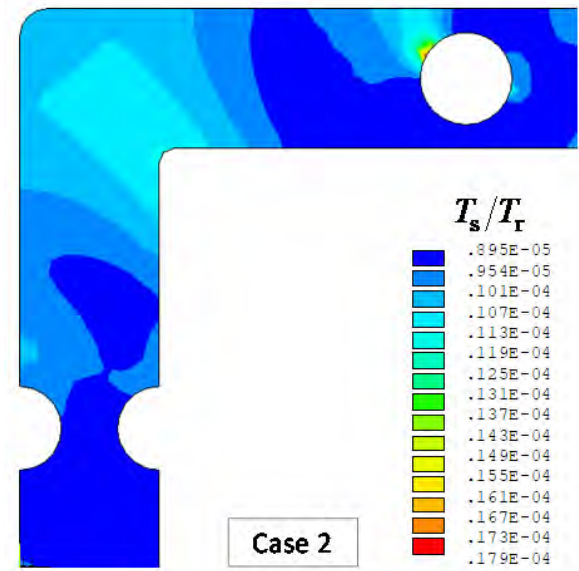

Fig. 9. Distribution of $T_{\mathrm{s}} / T_{\mathrm{r}}$ (Case 2).

This effect is further confirmed by considering the Case 3 , in which the input acceleration PSD is almost entirely centred on the first natural frequency. In this case, as can be seen in Table 1, the difference between Eq. (1) and (19) is largely increased.

On the other hand, one may presume that the observed discrepancies between the scaling law models are caused from taking the exponent $\alpha$ in Eq. (1) equal to the material inverse slope, instead of an "empirical" value around 3-5, as suggested in [3]. This conclusion is, however, not confirmed by the results in the last column of Table 1, which lists the values of $\alpha$ which would make Eq. (1) fit the time duration $T_{\mathrm{s}}$ resulting from the A-Z model (19), as implemented in numerical modelling. These values of $\alpha$ are largely dependent on the type of input acceleration PSD and it would then rather difficult to determine in advance a proper value of $\alpha$.

To overcome the above limitations of the simplified scaling law (1), the use of the Allegri-Zhang model preferably in its bandwidth corrected form (19) - is thus recommended. An example of output $\left(T_{\mathrm{s}} / T_{\mathrm{r}}\right)$ as given by Eq. (19) is shown in Fig. 9 for the Case 2.

\section{The role of S-N parameters}

According to the Palmgren-Miner rule, the fatigue parameters $b, C$ in the expressions presented so far should be those from the constant amplitude S-N curve. Experimental results, however, seem to highlight a change in fatigue parameters in random loading tests. In this case the S-N curve is generally located on the left of the corresponding S-N curve for constant amplitude loading, if the RMS value is used for comparison (limitations in the use of RMS are discussed in [8]). A higher $b$ is also observed in the random loading case. The shape of the stress PSD seems to have a limited effect [3,9].

A more important aspect is the role of tension and torsion S-N parameters in scaling laws, and especially with regard to the EVMS criterion adopted by A-Z method. It has been recognised (see [10]) that the EVMS criterion neglects the S-N parameters for torsion loading (in particular, it implicitly assumes that the torsion $\mathrm{S}-\mathrm{N}$ line is parallel to the tension one and exactly scaled by $\sqrt{ } 3$ ). These assumptions mean, for example, that Eq. (15) and (19) would return the same ratio of time durations, regardless of the torsion S-N curve parameters. Also note how the simple power law (1) does not even consider the torsion properties at all. Materials, on the other hand, are characterised by a wide range of torsion fatigue properties [10].

To account for both tension and torsion S-N parameters in the A-Z scaling law, other multiaxial spectral methods, among those currently available in the literature [11-13], should be used in place of the EVMS criterion.

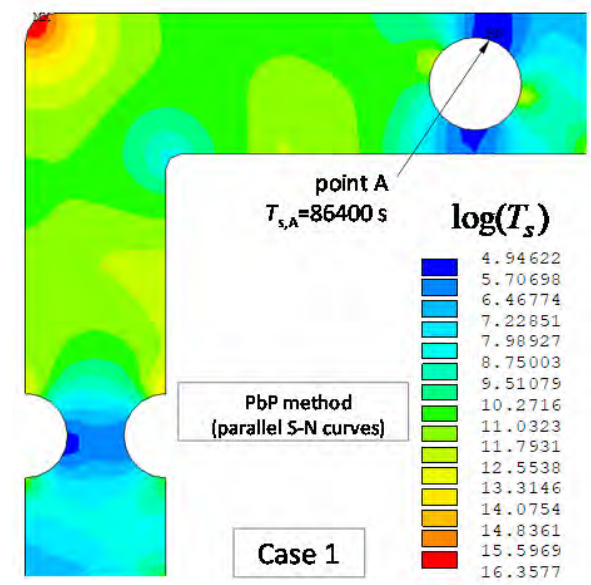

Fig. 10. Distribution of $\log \left(T_{\mathrm{s}}\right)$ (Case 1 ) by $\mathrm{PbP}$ criterion (material with parallel S-N lines).

A proposal is made here to reformulate the A-Z scaling law by using the so-called "Projection-by-Projection" $(\mathrm{PbP})$ criterion, which is a stress invariant-based multiaxial criterion that can account for both torsion and tension S-N parameters (the main theoretical aspects are outside the goal of this work and they can be found elsewhere [14,15]). The PbP method computes the fatigue damage in time $T$ as:

$$
D_{\mathrm{PbP}}=T\left[\sum_{\mathrm{i}=1}^{5} d_{\mathrm{i}}^{\frac{2}{k_{\mathrm{ref}}}}\right]^{\frac{k_{\text {ref }}}{2}}
$$

where $d_{\mathrm{i}}$ is the damage intensity (damage/s) of the $i$-th stress projection in the deviatoric space, which can be estimated by uniaxial spectral methods, see Section 3.1. If the time to failure (i.e. the inverse of fatigue damage) for each stress projection is estimated by the TB method for wide-band stress (see Eq. (8)), the following scaling law is obtained:

$$
\frac{T_{\mathrm{f}, \mathrm{s}}^{(\mathrm{e})}}{T_{\mathrm{f}, \mathrm{r}}^{(\mathrm{e})}}=\frac{C_{\mathrm{ref}, \mathrm{s}}^{(\mathrm{e})} \Gamma\left(1+\frac{b_{\mathrm{ref}, \mathrm{r}}^{(\mathrm{e})}}{2}\right)\left[\sum_{i=1}^{5}\left(\lambda_{0 \mathrm{i}, \mathrm{r}}^{(\mathrm{e})}\left(\rho_{\mathrm{i}, \mathrm{r}}^{(\mathrm{e})} v_{0 \mathrm{i}, \mathrm{r}}^{(\mathrm{e})}\right) \frac{2}{b_{\mathrm{ref}, \mathrm{r}}^{(\mathrm{e})}}\right)\right]^{\frac{b_{\mathrm{ref}, \mathrm{r}}^{(\mathrm{e})}}{2}}}{C_{\mathrm{ref}, \mathrm{r}}^{(\mathrm{e})} \Gamma\left(1+\frac{b_{\mathrm{ref}, \mathrm{s}}^{(\mathrm{e})}}{2}\right)\left[\sum_{i=1}^{5}\left(\lambda_{0 \mathrm{i}, \mathrm{s}}^{(\mathrm{e})}\left(\rho_{\mathrm{i}, \mathrm{s}}^{(\mathrm{e})} v_{0 \mathrm{i}, \mathrm{s}}^{(\mathrm{e})}\right) \frac{2}{b_{\mathrm{ref}, \mathrm{s}}^{(\mathrm{e})}}\right)\right]^{\frac{b_{\mathrm{ref}, \mathrm{s}}^{(\mathrm{e})}}{2}}}
$$


in which $\lambda_{0 \mathrm{i}}, v_{0 \mathrm{i}}, \rho_{\mathrm{i}}$ are the variance, zero up-crossing rate and bandwidth correction factor of the $i$-th stress projection. In Eq. (21), $C_{\text {ref }}$ and $b_{\text {ref }}$ are the parameters of a reference S-N curve in a Modified Wöhler Diagram, defined in the deviatoric space. They are linearly interpolated from the tension and torsion $\mathrm{S}-\mathrm{N}$ parameters (see $[14,15])$.

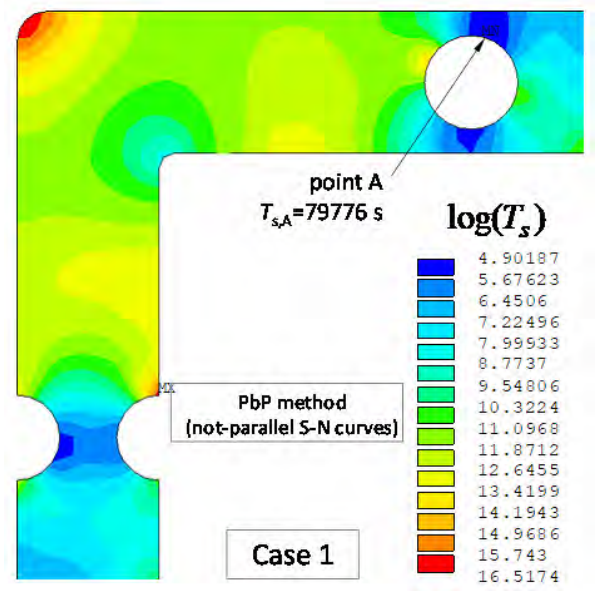

Fig. 11. Distribution of $\log \left(T_{\mathrm{s}}\right)$ (Case 1 ) by $\mathrm{PbP}$ criterion (material with non-parallel S-N lines).

To appreciate the effect of S-N parameters, Fig. 10 and Fig. 11 compare the distribution of $\log \left(T_{\mathrm{s}}\right)$ estimated by the $\mathrm{PbP}$ criterion, when considering two types of materials with: i) S-N curves parallel and scaled by $\sqrt{3}$ (Fig. 10), ii) none of the previous (Fig. 11). The figures refer to the Case 1 acceleration PSD. The comparison demonstrates that the $\mathrm{PbP}$ is sensitive to the torsion $\mathrm{S}-\mathrm{N}$ parameters and the time duration changes from material i) to material ii). The difference would be more pronounced if the input acceleration PSD were centred around a single vibration mode characterised by a torsional deformation. By contrast, no difference would be observed in the time duration for material i) and ii), if the EVMS were used in the A-Z solution, see Eq. (15) or (19). In both cases, the distribution of $\log \left(T_{\mathrm{s}}\right)$ would be that shown in Fig. 7.

\section{Conclusions}

This work dealt with inverse scaling laws used to calibrate accelerated vibratory tests for linear systems undergoing stationary Gaussian excitations. A comparison was made between a simple inverse power law model and the Allegri-Zhang (A-Z) solution, which was first reviewed and next modified by introducing a bandwidth correction factor to account for the actual shape of the frequency spectrum for the "equivalent von Mises stress" (EVMS).

A numerical example (L-beam submitted to random base accelerations) solved by the finite element method was used as a test case for the comparison. Several types of idealised rectangular acceleration PSDs for the laboratory environment were considered, to investigate the sensitivity of the scaling law to the type of input.
The results confirmed that both scaling laws agree only for "real" and "lab" acceleration PSDs which are exactly scaled. In the other cases, large differences occur and the power law model estimates a much longer time duration than the A-Z solution. This difference was explained in terms of the influence of the system dynamics on the time duration estimated by numerical analysis, which instead is ignored by the simple power law model. Numerical results also showed that the values of the exponent $\alpha$ in the power law model largely dependent on the type of acceleration PSD; choosing a priori a suitable value of $\alpha$ would then be rather difficult.

An attempt was finally made to reformulate the A-Z scaling law in terms of the "Projection-by-Projection" multiaxial spectral method, in place of the EVMS criterion. This modification permitted the A-Z solution to be sensitive also to the torsion S-N properties.

In summary, the results presented in this work emphasised the limitations in the use of the simple power law model and they suggested that the A-Z solution should be preferred.

\section{References}

1. MIL-STD-810 CF, Military standard environmental test methods for aerospace and ground equipment, Method 514: Vibration (1962)

2. A. Angeli, B. Cornelis, M. Troncossi, Mech. Systems and Signal Proc. 103, 340 (2018)

3. G. Allegri, X. Zhang, Int. J. Fatigue 30(6), 967 (2008)

4. L.D. Lutes, S. Sarkani, Random vibrations: analysis of structural and mechanical systems (Elsevier, USA, 2004)

5. D. Benasciutti, R. Tovo, Int. J. Fatigue 27(8), 867 (2005)

6. D. Benasciutti, R. Tovo, Probab. Eng. Mech. 21(4), 287 (2006)

7. X. Pitoiset, A. Preumont, Int. J. Fatigue 22, 541 (2000)

8. C.M. Sonsino, Int. J. Fatigue 11(3), 142 (1989)

9. C. Lalanne, Mechanical Vibration and Shock Analysis - vol. 4: Fatigue Damage (ISTE Ltd, UK, 2009)

10. D. Benasciutti, J. Sound Vib. 333(18), 4326 (2014)

11. D. Benasciutti, F. Sherratt, A. Cristofori, Procedia Engineering 101(C), 34 (2015)

12. D. Benasciutti, F. Sherratt, A. Cristofori, Int. J. Fatigue 91(2), 397 (2016)

13. K. Bel Knani, D. Benasciutti, A. Signorini, R. Tovo, Int. J. Mater. Prod. Technol. 30(1-3), 172 (2007)

14. A. Cristofori, D. Benasciutti, R. Tovo, Int. J. Fatigue 33(7), 887 (2011)

15. A. Cristofori, D. Benasciutti, SAE Technical Paper 2014-01-0924 (2014) 